\title{
Biologic therapy for advanced breast cancer: recent advances and future directions
}

\author{
Paolo Tarantino ${ }^{\mathrm{a}, \mathrm{b}}$, Stefania Morganti ${ }^{\mathrm{a}, \mathrm{b}}$ and Giuseppe Curigliano ${ }^{\mathrm{a}, \mathrm{b}}$

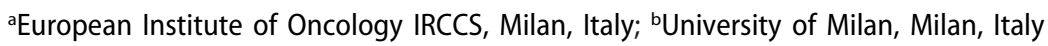

ABSTRACT

Introduction: Advanced breast cancer $(A B C)$ is a leading cause of mortality, morbidity, and disability in women worldwide. For decades, treatment of $A B C$ has relied on chemotherapy and endocrine treatments (ET), until HER2 was recognized as a 'druggable' target in the 1990s. Thereafter, various anti-HER2 drugs have been approved for the HER2-positive subtype, but only in the last few years, biologic agents targeting different pathways have entered the therapeutic arsenal of luminal and triple-negative cancers.

Areas covered: The purpose of the present review is to recapitulate the most promising novel biologic agents being developed for the treatment of $A B C$. New drugs for all breast cancer subtypes are discussed, as well as some potential future directions in $A B C$ treatment.

Expert opinion: Several biologic drugs have been recently approved, revolutionizing $A B C$ treatment algorithms: key examples are CDK4/6-inhibitors and the PI3K-inhibitor alpelisib for endocrine-positive ABC; atezolizumab for triple-negative cancers; two PARP-inhibitors for HER2-negative germinal BRCAmutated cancers. Additionally, multiple drugs are demonstrating activity in late-phase clinical trials for all subtypes. While some of these represent pharmacological evolutions of previously approved drugs, some others might pave the way for new paradigms in $A B C$, challenging both its classification and current treatment algorithms.
ARTICLE HISTORY

Received 4 November 2019

Accepted 2 April 2020

KEYWORDS

Antibody-drug conjugates; breast cancer; CDK4/6 inhibitors; HER2-low; immunotherapy; new drugs; PARP inhibitors; precision medicine

\section{Introduction}

For decades, the treatment of advanced breast cancer (ABC) has predominantly consisted of traditional chemotherapies and endocrine treatments (ET). One relevant step forward was achieved in the 1990s, with the development and approval of the first biological agent, the anti-HER2 monoclonal antibody (mAb) trastuzumab [1]. Since then, several other anti-HER2 agents have been approved for the subgroup of HER2-positive $A B C$ [2], while treatment of luminal HER2negative and triple-negative cancers has kept consisting mainly of ET and chemotherapies. However, in the last few years, various new biological agents have entered the clinical practice in all subgroups of breast cancer (BC) (Figure 1). Three CDK4/6-inhibitors have been approved for the treatment of hormone receptor-positive $A B C$, showing to improve overall survival (OS) in this population [3-5]. Alpelisib has been approved for PIK3CA-mutated luminal $A B C$, in combination with ET $[6,7]$. The PARP-inhibitors olaparib and talazoparib have been approved for the treatment of HER2-negative germinal BRCA1/2-mutated (gBRCA-mut) $A B C$, regardless of hormone receptor expression, based on the benefit showed by two independent randomized controlled phase 3 clinical trials. Finally, the anti-PDL1 mAb atezolizumab has been positioned in the management of PDL1-positive advanced triplenegative breast cancer (TNBC). Drug development in this field has kept increasing its pace, and multiple highly active therapies are under study for the treatment of all $A B C$ subgroups. The purpose of the present review is to recapitulate the most promising biological agents currently under early- and latestage development and to underline the future directions in which $A B C$ drug development is moving.

\section{Hormone receptor-positive breast cancer}

Estrogen receptor (ER) represents the first target discovered in the history of breast cancer [8]. Since the approval of tamoxifen in the 1970s, several ET have been added in the therapeutic armamentarium of luminal BC, including Selective Estrogen Receptor Modulators (SERMs), Selective Estrogen Receptor Downregulators (SERDs), and Aromatase Inhibitors (Als) [9].

These agents have represented the cornerstone of luminal $A B C$ treatment for decades, prolonging survival and sparing toxicity of chemotherapy to many women. Despite this fact, almost all $A B C s$ ultimately lose responsiveness to hormonal treatments, and many efforts are ongoing to identify strategies to overcome this resistance.

Activation of alternative intracellular pathways is a recognized resistance mechanism to ET [10]. Several different biological agents targeting these pathways have demonstrated to reverse endocrine sensitivity when combined to hormonal drugs.

\subsection{CDK $4 / 6$ inhibitors}

CDK4/6-inhibitors are selective inhibitors of cyclin-dependent kinases 4 and 6, enzymes directly involved in the regulation of cell cycle transition from G1- to S-phase. When combined with 


\section{Article highlights}

- In the last 20 years, the introduction of biologic drugs has progressively revolutionized treatment algorithms of all breast cancer subtypes

- More recently, an accelerated pace in biologic drug approval in breast cancer has been observed, with about half of all approvals taking place in the last 3 years

- Novel anti-HER2 antibody-drug conjugates are challenging traditional treatment paradigms, showing activity in the emerging entity of HER2-low breast cancers

- As more genetic alterations become actionable, molecular profiling of $A B C$ becomes increasingly important for the optimal selection of treatments

- Biologic treatments inevitably increase treatment costs, and a major challenge in the future will be ensuring optimal accessibility to novel treatments in developing countries

This box summarizes key points contained in the article.

fulvestrant or Als, these agents have demonstrated to improve treatment outcomes, with a favorable toxicity profile.

The MONALEESA-2 [11,12], PALOMA-2 [13,14] and MONARCH-3 $[15,16]$ trials, respectively, tested ribociclib, palbociclib, and abemaciclib in combination with Als as first-line treatment for luminal BC. Similarly, the PALOMA-3 $[5,17]$ and MONARCH-2 [18] trials combined palbociclib and abemaciclib with fulvestrant in patients who had previously progressed on ET alone, while the MONALEESA-3 study tested the combination of ribociclib and fulvestrant in both treatment-naïve and women progressed to up to one line of ET [19]. All of these studies reached the primary endpoint, with a significant PFS increase, and CDK4/6-inhibitors + ET became standard of care for luminal $A B C$ as both first-line treatment or after progression to a previous ET. Additionally, ribociclib was tested in combination with $\mathrm{Al}$ or tamoxifen (plus goserelin) in premenopausal women in the phase 3 MONALEESA-7 trial, showing a relevant and statistically significant OS improvement in the study cohort [20].

Results in terms of OS of CDK4/6-inhibitors plus fulvestrant have been recently presented: two of these trials have reached statistical significance (MONALEESA-3 [21], MONARCH-2 [4]), whereas the PALOMA-3 [5] showed a strong trend. OS data in first line are maturing.

All of these agents were well tolerated, with slightly different toxicity profiles [22]. Most frequent observed adverse events (AEs) across trials were neutropenia for palbociclib and ribociclib, diarrhea for abemaciclib, liver toxicity for ribociblib and abemaciclib, and QT interval prolongation for ribociclib. Interestingly, despite the common occurrence of neutropenia with these agents, febrile neutropenia was a relatively rare event in all trials, occurring in less than $2 \%$ of the patients.

The best strategy after progression on CDK4/6-inhibitors and ET still needs to be defined. Chemotherapy should be avoided in patients without visceral crisis, but ET alone may not be active enough. Some trials are exploring the efficacy of continuing CDK4/6-inhibitors beyond progression with a different ET, whereas others are combining CDK4/6-inhibitors with biological agents targeting other pathways and/or immunotherapy (Table 1). Alternative strategies are testing these new agents plus CDK4/6-inhibitors and ET in the same setting of approval trial, to verify if a triple combination can further increase outcomes.

\subsection{PI3K/AKT/mTOR pathway}

The PI3K/AKT/mTOR pathway is frequently deregulated in BC [23]. Drugs targeting this pathway have been deeply investigated, with contradictory results.

Everolimus, an mTOR-inhibitor, is the first biological agent approved for luminal ABC. The BOLERO-2 [24] trial showed a PFS improvement with the combination of exemestane and everolimus versus exemestane alone, with a median PFS (mPFS) of 6.9 versus 2.8 months (hazard ratio [HR] 0.43; 95\% confidence interval [Cl], 0.35-0.54; $\mathrm{p}<0.001)$, even if toxicity was higher in the experimental arm. In particular, treatment with everolimus resulted in a higher rate of grade 3-4 stomatitis, anemia, hyperglycemia, and pneumonitis. Due to the challenging toxicity profile, the compound is still only partially implemented in clinical practice.

Alpelisib is an oral a-selective inhibitor of PI3K. The phase 3 SOLAR-1 [6] trial compared the combination of fulvestrant \pm alpelisib in patients with ET-pretreated luminal ABC. mPFS was significantly higher with the combination therapy (11 vs 5.7 months; HR $0.65 ; 95 \% \mathrm{Cl}, 0.50$ to $0.85 ; \mathrm{p}<0.001$ ) in patients with PI3KCA-mutated tumors, whereas this advantage was not observed for PI3KCA-wild type tumors. Most frequent $\geq \mathrm{G} 3 \mathrm{AEs}$ were hyperglycemia ( $36.6 \%$ vs $0.7 \%$ in experimental vs standard arm), rash ( $20.1 \%$ vs $0.3 \%)$, and diarrhea (6.7\% vs $0.3 \%)$. The results of this trial led to alpelisib FDA-approval for PI3KCA-mutated luminal $A B C$, whereas approval is still pending in Europe.

Since only a minority of patients enrolled in the SOLAR-1 was pretreated with CDK4/6-inhibitors (6\%), the BYLieve trial

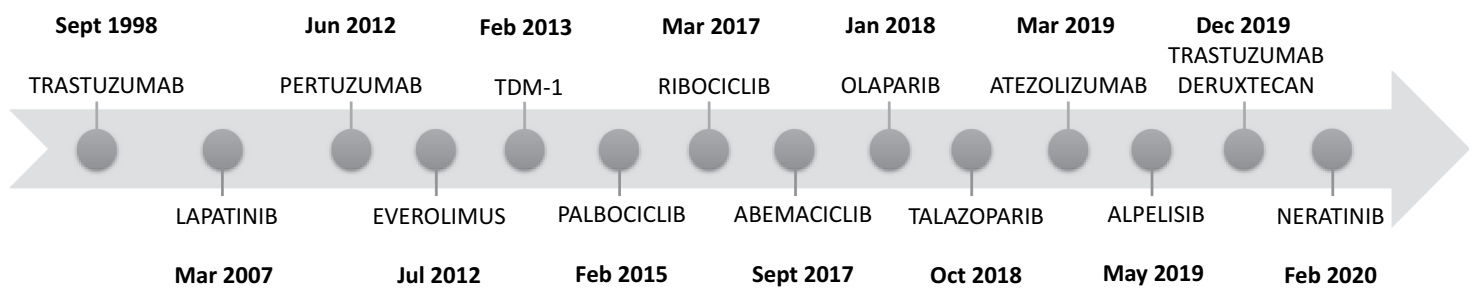

Figure 1. Timeline of FDA-approval biologic treatments for $A B C$ management.

Since the approval of the first biologic drug in ABC treatment (1998), drug development in this field has kept increasing its pace, with half of all the approvals taking place between 2017 and 2019. 
EXPERT OPINION ON BIOLOGICAL THERAPY

3

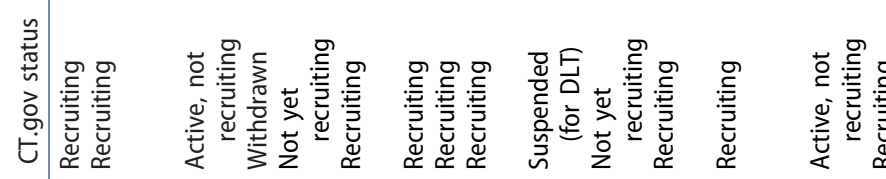

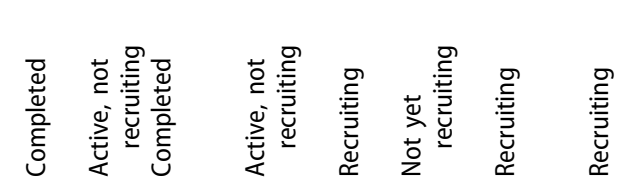

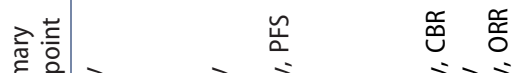

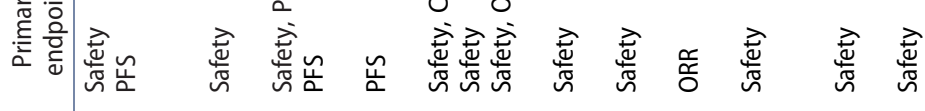

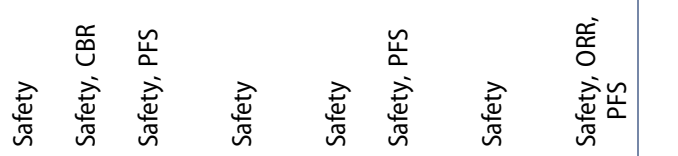

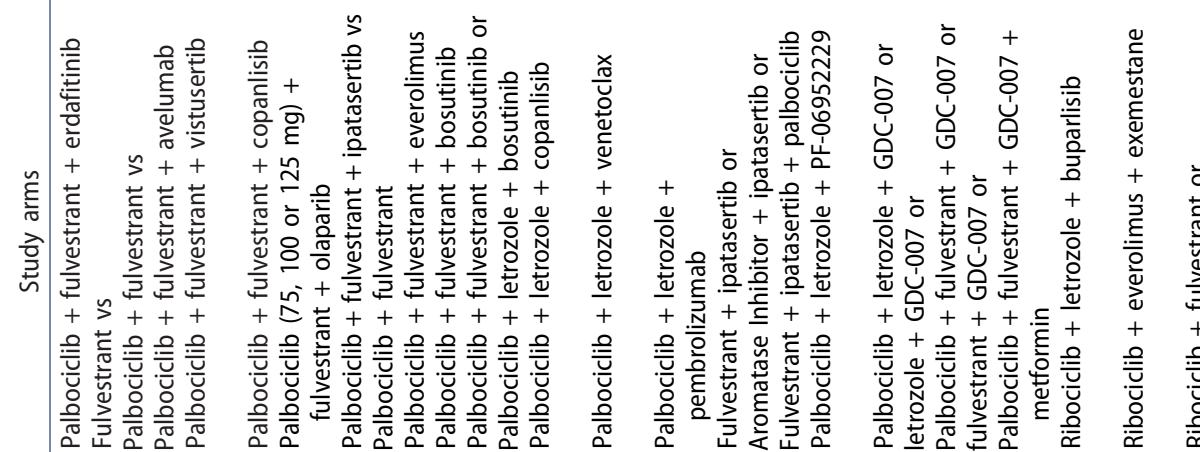

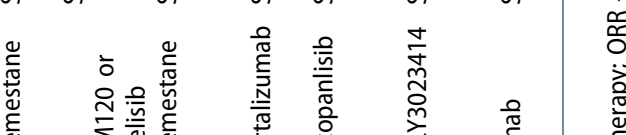

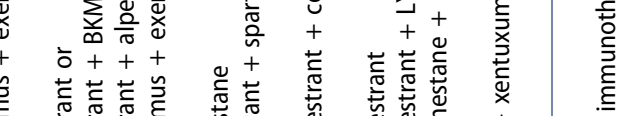

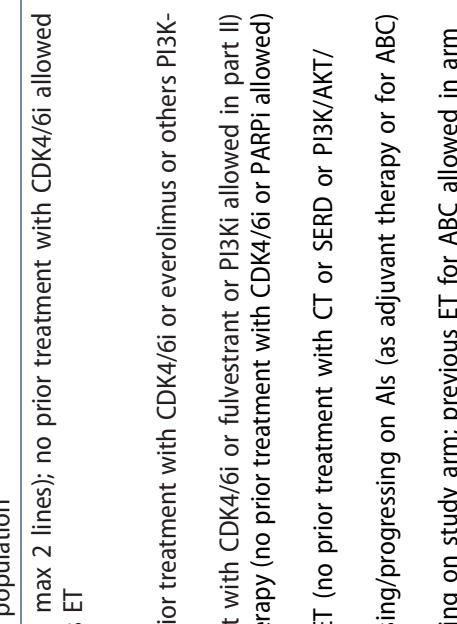

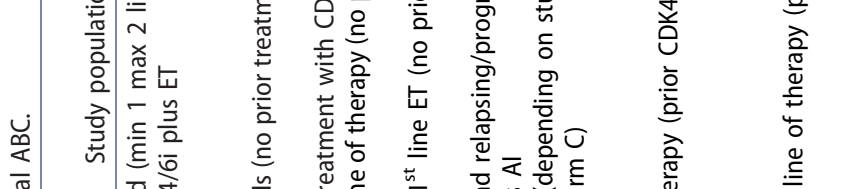

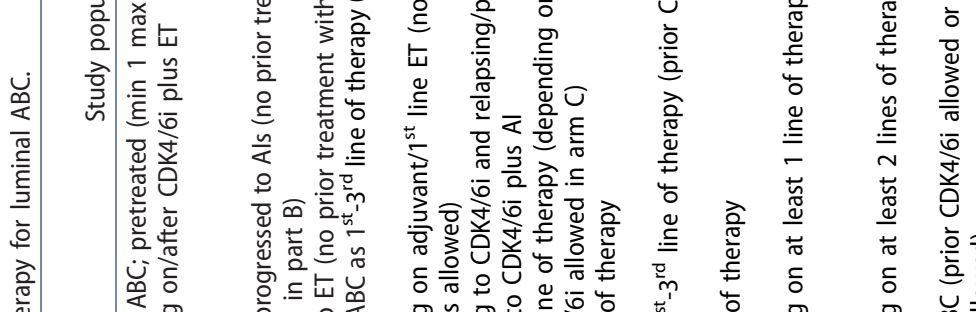

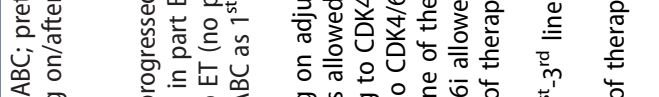

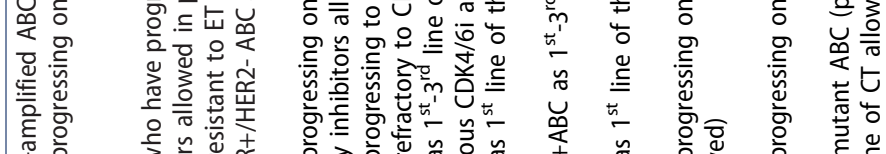

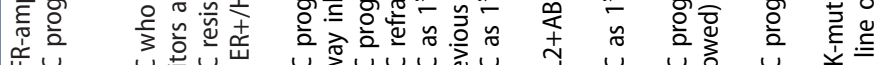

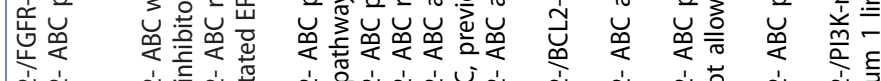

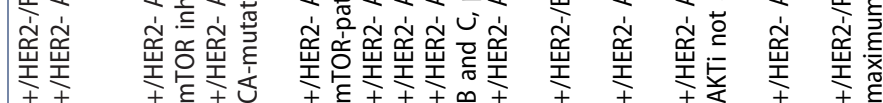

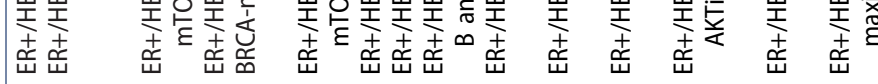

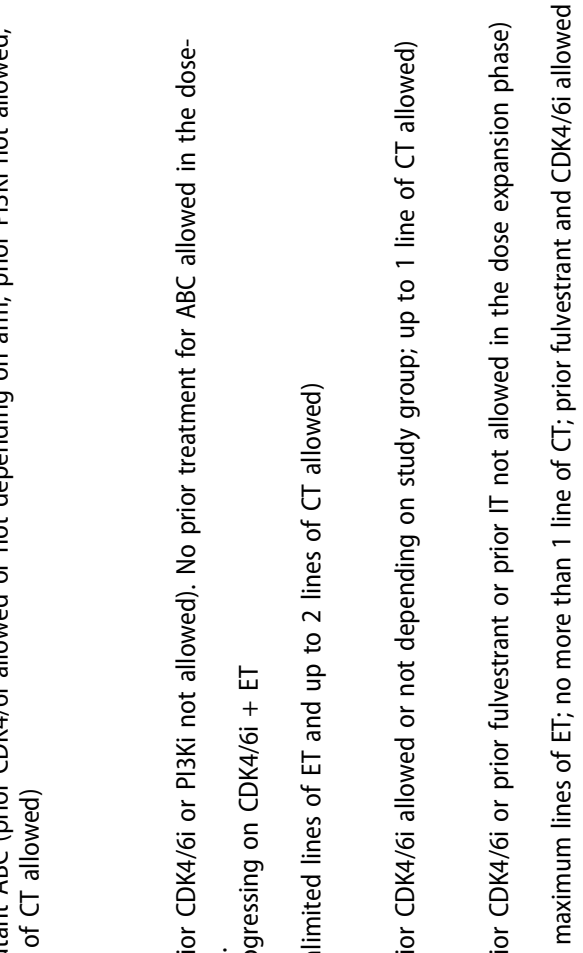

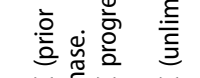

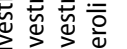

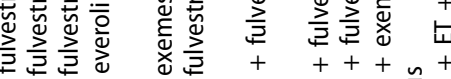

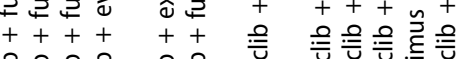

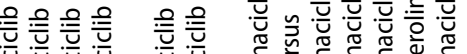

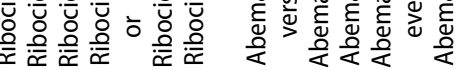

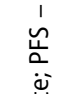

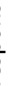


(NCT03056755) was initiated, to test the efficacy of alpelisib plus ET after progressing on CDK4/6-inhibitors + ET.

Taselisib is a selective PI3K-inhibitor (PI3Ki) targeting alpha, delta, and gamma isoforms. The combination of taselisib plus fulvestrant was investigated versus fulvestrant alone in the phase 3 SANDPIPER trial [25], showing a significant but small mPFS increase in the combination arm (mPFS 7.4 vs 5.4 months; HR 0.70; $95 \% \mathrm{Cl}$ not provided; $\mathrm{p}<0.01$ ). Nevertheless, toxicity was important, leading to more taselisib discontinuations ( $17 \%$ vs $2 \%$ ) and dose reductions ( $37 \%$ vs $2 \%)$, versus placebo. A phase 2 trial testing taselisib plus tamoxifen is ongoing (POSEIDON trial).

Buparlisib is a highly selective pan-class I PI3K inhibitor tested in combination with several ET. Preliminary results from the phase 2 BELLE-2 trial [26] showed a significant PFS improvement from the combination of buparlisib plus fulvestrant versus fulvestrant alone (6.9 vs 5 months; $\mathrm{HR} 0.78 ; 95 \% \mathrm{Cl}$ 0.67-0.89; $\mathrm{p}<0.001$ ), with a favorable trend in OS (HR 0.87; $95 \% \mathrm{Cl} 0.74-1.02 ; \mathrm{p}=0.045)$ [27]. These results were confirmed in phase 3 BELLE-3 study [28], where mPFS was significantly higher in the combination arm (3.9 vs 1.8 months; HR 0.67, 95\% Cl 0.53-0.84; $\mathrm{p}<0.01$ ). Despite these results, the clinical development of buparlisib was stopped because of excessive toxicities. As high as $61 \%$ of patients in the buparlisib group experienced $\geq \mathrm{G} 3$ AEs, with elevated aminotransferases, hyperglycemia, and hypertension being the most frequent.

Capivasertib is an orally available pan-AKT inhibitor, for which efficacy data have been recently published. The phase 2 FAKTION trial [29] enrolled patients with luminal ABC pretreated with $\mathrm{Al}$, randomizing them to receive fulvestrant with or without capivasertib. Patients were stratified according to PIK3CA and PTEN status. mPFS was 10.3 months for capivasertib versus 4.8 months for placebo (HR 0.58; 95\% Cl 0.39-$0.84 ; \mathrm{p}<0.01$ ), and median OS was 26 months for capivasertib compared to 20 months for placebo (HR 0.59; 95\% Cl 0.34-1.05; $p=0.071)$. However, the combination of fulvestrant and capivasertib in AKT-mutated cancers was not investigated in this trial. The most common $\geq \mathrm{G} 3$ AEs were hypertension (32\% vs $24 \%$ ), diarrhea ( $14 \%$ vs $4 \%$ ), and fatigue (1 vs $4 \%$ ), and two deaths were possibly related to the study regimen.

\subsection{Histone deacetylase inhibitors}

Epigenetic modifications are often implied in endocrineresistance in $A B C$ [30]. To overcome such resistance mechanism, several histone deacetylase (HDAC) inhibitors are under investigation.

Entinostat is a selective, oral, class I HDAC inhibitor. In luminal $A B C$, the combination of entinostat and exemestane has demonstrated to significantly improve mPFS versus exemestane alone in a phase 2 trial [31] (4.28 vs 2.27 months, HR $0.73 ; 95 \% \mathrm{Cl}$ not reported; $\mathrm{p}=0.06$ ). A randomized phase 3 trial testing the same combination is ongoing.

Tucidinostat is a subtype-selective HDAC-inhibitor entirely developed in China and already approved in this country. The phase 3 ACE trial [32] tested exemestane \pm tucidinostat in ETresistant postmenopausal patients, showing a significant PFS improvement (7.4 vs 3.8 months; HR 0.75; 95\% Cl 0.58-0.98; $p=0.033$ ). Nevertheless toxicities were not negligible, and $48 \%$ of treatment interruptions were recorded in the experimental arm. Most frequently observed AEs were hematological, hypokalemia, and nausea.

\subsection{FGFR-inhibitors}

Fibroblast Growth Factor Receptor (FGFR) amplifications are detected in about $14 \%$ of $B C$, mainly in the luminal subtype [33]. FGFR-inhibition in luminal BC has been tested with both selective FGFR-inhibitors and multitarget tyrosine kinase inhibitors (TKI) with additional anti-angiogenic action.

AZD4547 is a highly selective inhibitor of FGFR1-3. The phase 1b/2a RADICAL trial [34] tested AZD4547 in combination with Als, showing preliminary activity and safety in an FGFR-unselected population. The phase 2 GLOW trial instead enrolled only patients with FGFR1 polysomy or gene amplification, randomizing them to receive fulvestrant \pm AZD4547. Data from this trial are still awaited.

Lucitanib is a dual inhibitor targeting VEGFR 1-3 and FGFR $1-3$. The phase $1 \mathrm{~b}$ INES trial [35] tested lucitanib in combination with fulvestrant in FGFR-unselected fulvestrant-pretreated luminal $A B C$, with some signals of activity (ORR 16.7\%) but an unfavorable safety profile (high rate of G3-4 hypertension and fatigue). Further data were reported from the phase 2 FINESSE trial [36], testing lucitanib in FGFR1-altered and wild-type ERpositive/HER2-negative patients. Overall, 76 patients were enrolled, with modest activity (ORR 19\%) demonstrated only in FGFR1 amplified patients, and significant cardiovascular toxicities ( $\geq \mathrm{G} 3$ hypertension in $66 \%$ of patients) related to the anti-angiogenic effect of lucitanib.

\section{HER2-positive breast cancer}

Since the approval of trastuzumab in 1998, several other antiHER2 agents have been developed for HER2-positive ABC treatment, across diverse pharmacological classes. Until 2019, four of these agents had been FDA and/or EMA approved for the treatment of $A B C$ : the $m A b s$ trastuzumab and pertuzumab, the TKI lapatinib and the antibody-drug-conjugate (ADC) trastuzumab emtansine (TDM-1) [2]. Novel agents belonging to all of these classes are under development, and new agents with different mechanisms of action are showing activity in this context. In particular, novel anti-HER2 TKIs are showing encouraging results in combination with chemotherapy, especially in patients with central nervous system (CNS) disease, and novel ADCs are demonstrating impressive activity in highly pretreated HER2-positive $A B C$ patients. Of note, the latter class of agents is showing potential activity in the treatment of HER2-low ABC, a wide category of patients for which no anti-HER2 agent has ever demonstrated activity. Such results are likely related to engineering improvements leading to the synthesis of ADCs with higher drug-to-antibody ratio (DAR) and cleavable linkers. These features allow the so-called bystander killing effect, namely the activity of the compound not only against cancer cells expressing the target but also against surrounding cells. 


\subsection{Anti-HER2 monoclonal antibodies}

Margetuximab is an Fc-engineered anti-HER2 mAb, designed to increase affinity for the activating $\mathrm{Fc}$ receptor CD16A and decrease affinity for the inhibitory Fc receptor CD32B. Preliminary results from the randomized phase 3 SOPHIA trial of margetuximab + chemotherapy in pretreated HER2-positive (both hormone receptor-positive and -negative) $A B C$ demonstrated a statistically significant improvement in PFS over trastuzumab + chemotherapy (mPFS 5.8 vs 4.9 months; HR $0.76 ; 95 \% \mathrm{Cl} 0.59-0.98 \mathrm{p}=0.033)$, which was more pronounced in patients with CD16A genotypes containing a $158 \mathrm{~F}$ allele (mPFS 6.9 vs 5.1 months; HR $0.68 ; 95 \% \mathrm{Cl}$ $0.52-0.90 ; p=0.005$ ) [37]. A preliminary analysis of OS was also recently reported, with an HR of 0.95 in the overall population (95\% Cl $0.69-1.31)$, and an HR of 0.82 for the genotype-restricted population $(95 \%$ Cl $0.58-1.17)$ [38]. Safety was comparable in both arms. OS data are still maturing, and will potentially clarify the ultimate clinical impact of the compound.

\subsection{Anti-HER2 tyrosine kinase inhibitors}

Neratinib is an irreversible pan-HER TKI, currently approved for the extended adjuvant treatment of HER2-positive early $B C$ based on the results of the phase 3 ExteNET trial [39]. Various trials have tested the drug in the advanced setting. In the phase 2 NEfERT-T trial, neratinib failed to show a superior activity compared with trastuzumab when associated to paclitaxel, although a superior CNS activity was noted [40]. A promising CNS activity was also shown by the phase 2 TBCRC022 trial in ABC patients with brain metastases, with a CNS response rate ranging between $33 \%$ and $49 \%$ depending on previous TKI treatment [41]. Finally, data from the randomized phase 3 NALA trial were recently presented: compared with lapatinib and capecitabine, neratinib and capecitabine demonstrated an improved activity in terms of PFS, with a similar toxicity profile [42]. Overall, the main toxicity identified was diarrhea, $\geq G 3$ in about $30 \%$ of the patients across the trials. Following these results, FDA approved the combination of neratinib and capecitabine for HER2-positive ABC pretreated with $\geq$ prior anti-HER2-based treatments.

Tucatinib is a HER2-selective TKI currently in the study for $A B C$, for which a promising activity was reported in early phase trials. In a phase $1 \mathrm{~b}$ trial testing the combination of tucatinib + TDM1 in second line after trastuzumab and a taxane, an ORR of $48 \%$ was reported, with an acceptable toxicity profile [43]. A further phase $1 \mathrm{~b}$ trial tested tucatinib in combination with trastuzumab and capecitabine, finding an ORR of $61 \%$ and a brain-specific ORR of $42 \%$, with fewer EGFRrelated AEs compared with other anti-HER2 TKIs [44]. These results guided the design of the randomized phase 2 HER2CLIMB trial, which tested the combination of capecitabine and trastuzumab with or without tucatinib in TDM1pretreated HER2-positive ABC patients [45]. Results from this trial were recently published, showing a statistically significant improvement of PFS at 1 year from $12.3 \%$ to $33.1 \%$ (HR 0.54; $95 \% \mathrm{Cl} 0.42$ to $0.71 ; \mathrm{p}<0.001)$ and a statistically significant improvement in OS from 17.4 months to 21.9 months in the study arm (HR 0.66; $95 \% \mathrm{Cl} 0.50$ to $0.88 ; \mathrm{p}=0.005$ ) [46]. ORR was also improved in the tucatinib arm, and the drug demonstrated a remarkable activity also in patients with brain metastasis at enrollment. There was a higher rate of $\geq \mathrm{G} 3$ diarrhea $(12.9 \%$ vs $8.6 \%)$ and aminotransferase increase $(5 \%$ vs $0.5 \%)$ in the study arm, with most of other toxicities being comparable. Based on these results, the compound has been recently granted Priority Review by FDA.

Poziotinib is an irreversible pan-HER TKI which has shown interesting activity for pretreated HER2-positive ABC patients. In a phase 2 trial conducted in South Korea, the compound achieved an ORR of $25 \%$ and a mPFS of 4 months, with diarrhea, stomatitis, and rash being the most common AEs [47]. Further trials are testing the compound in combination with TDM1 or in patients harboring EGFR/AR alterations.

Pyrotinib is an irreversible pan-HER TKI for which encouraging data has recently been reported in pretreated HER2positive $A B C$. A phase 3 trial conducted in China randomized 279 HER2-positive $A B C$ patients to receive capecitabine \pm pyrotinib, finding an ORR ( $68 \%$ vs $16 \%$ ) and PFS advantage in the pyrotinib arm (11 vs 4 months; HR $0.18 ; 95 \% \mathrm{Cl} 0.13-$ $0.26 ; p<0.001$ ) [48]. Furthermore, in a phase 2 trial enrolling 128 HER2-positive ABC patients, the capecitabine + pyrotinib combination achieved a higher ORR ( $78 \%$ vs $57 \%, \mathrm{p} 0.01)$ and PFS (18 vs 7 months; HR $0.36 ; 95 \% \mathrm{Cl}, 0.23-0.58 ; \mathrm{p}<0.01$ ) compared with capecitabine + lapatinib, with a comparable safety profile [49]. The randomized phase 3 PHOEBE is currently testing the same combinations on a larger study population.

\subsection{Anti-HER2 antibody-drug conjugates}

Trastuzumab deruxtecan is a HER2-targeting mAb conjugated with a topoisomerase I inhibitor (DXd), characterized by a high DAR (7-8) and an enzymatically cleavable linker, which enables an effective bystander effect. A single-group phase 2 trial tested the compound in 184 highly pretreated (median of 6 prior lines) HER2-positive ABC patients, showing an impressive ORR of $60.9 \%$, a DCR of $97.3 \%$, and a mPFS of 16.4 months [50]. Efficacy was seen in all patient subgroups, including patients with CNS disease, ER+ disease, and prior treatment with TDM1. Estimated OS at 1 year was $86 \%$, while mOS was not reached. The main $\geq \mathrm{G} 3$ toxicities were neutropenia $(20.7 \%)$, anemia $(8.7 \%)$, and nausea $(7.6 \%)$, likely related to the chemotherapy backbone. However, interstitial lung disease (ILD) emerged as a potentially severe $A E$, with $13.6 \%$ of the patients experiencing any-grade ILD, and four deaths related to the toxicity. Following the report of fatal cases of ILD, a robust monitoring and management plan has been established for all trastuzumab deruxtecan studies, with prompt detection and treatment of ILD, and study treatment discontinuation in symptomatic cases.

Of note, the compound showed relevant activity also in HER2-low patients (HER2 IHC 1+ or 2+ with negative ISH assay), a subgroup of patients for whom no anti-HER2 therapy is currently approved. Indeed, the conjugate was tested in a phase 1 trial enrolling 54 highly pretreated HER2-low ABC patients, finding an ORR of $37 \%$, a mPFS of 11.1 months, and 
a mOS of 29.4 months [51]. According to the promising activity seen in early phase trials, three phase 3 trials have been initiated, one of which in HER2-low patients [52]; moreover, two phase $1 \mathrm{~b}$ trials are testing the drug in combination with anti-PD1 antibodies. However, the impressive data observed in the abovementioned phase 2 trial were sufficient to prompt FDA accelerated approval of trastuzumab deruxtecan for pretreated HER2-positive ABC patients in early 2020.

Trastuzumab duocarmazine is a HER2-targeting mAb conjugated to a potent duocarmycin payload (vc-seco-DUBA) through a cleavable linker, with a DAR of 2.8. Results from the phase 1 trial testing the compound in multiple HER2expressing solid tumor patients revealed a promising ORR of $33 \%$ and mPFS of 7.6 months in HER2-positive $A B C$ patients [53]. BC patients were highly pretreated, with a median of 6 previous treatments. In analogy with trastuzumab deruxtecan, trastuzumab duocarmazine showed activity also in HER2-low ABC, with an ORR ranging between $28 \%$ and $40 \%$ depending on hormone receptors status [54]. $\geq \mathrm{G} 3$ toxicities occurred in $35 \%$ of the patients, mainly consisting of neutropenia, fatigue, and conjunctivitis. The phase 3 TULIP trial is currently ongoing, comparing trastuzumab duocarmazine to treatment of physician choice in HER2-positive BC.

\subsection{Anti-HER2 bispecific antibodies}

ZW25 is a bispecific/biparatopic antibody targeting two different domains of HER2 (ECD2/ECD4). After several in vivo studies demonstrating activity of the compound in both HER2positive and HER2-low expressing models, a phase 1 trial was initiated. Seventeen highly pretreated HER2-positive $A B C$ patients were enrolled, with 13 being evaluable for response [55]. In this cohort, PR rate was $46 \%$, with a DCR of $54 \%$. Toxicity profile was manageable, with only G1-2 AEs consisting of diarrhea and infusion reactions. Notably, by linking an auristatin payload to ZW25, the new compound ZW49 was derived, combining the mechanisms of action of ADCs and bispecific antibodies. The antibody is currently being tested in a phase 1 trial (NCT03821233)

\subsection{Immunotherapy in HER2-positive breast cancer}

Together with demonstrating a critical role in TNBC, immune checkpoint inhibitors (ICPI) are being tested in HER2-positive disease, with some signals of activity. The phase $1 \mathrm{~b} / 2$ PANACEA trial [56] tested the combination of trastuzumab and pembrolizumab in pretreated patients with HER2+ ABC. An ORR of $15 \%$ was achieved in the PDL1-positive population, whereas no responses were observed among PD-L1 negative patients. mPFS was similar between the two groups (2.7 and 2.5 months, respectively). OS data are still immature, but preliminary results underlined a possible benefit for patients with PDL1-positive tumors.

The KATE2 trial $[57,58]$ investigated instead the combination of TDM1 plus atezolizumab/placebo, identifying a possible treatment benefit restricted to PDL1-positive tumors (mPFS 8.5 vs 4.1 months; HR 0.60; $95 \% \mathrm{Cl} 0.32-1.11$; mOS not reached in both arms; HR 0.55; 95\% Cl 0.22-1.38). Several other trials combining
anti-HER2 agents and ICPI are ongoing, also in combination with chemotherapy as first-line treatment (NCT03125928).

\subsection{CDK4/6-inhibitors}

Various CKD4/6-inhibitors are being investigated in different combination for HER2-positive BC (Table 2). Indeed, preclinical evidence show that CDK4/6-inhibitors could result synergic with HER2-inhibition [59], together with reverting resistance to anti-HER2 agents [60]. Relevant data with two of these combinations have been recently reported.

Palbociclib was tested in combination with trastuzumab \pm letrozole in the SOLTI-PATRICIA phase 2 study, enrolling pretreated HER2+ patients [61]. At a preliminary analysis on 45 patients, the combination showed to be safe and active, particularly in the luminal subtype by PAM50, with a better clinical benefit rate (73\% vs $31 \%$ ) and mPFS (12.4 vs 4.1 months, HR $0.37 ; 95 \% \mathrm{Cl}$ not reported; $p$ 0.052) compared with nonluminal tumors.

Abemaciclib was studied in combination with trastuzumab and fulvestrant in the phase 2 MonarcHER trial for pretreated triple-positive (hormone receptor-positive/HER2-positive) patients [62]. At a recent report on 237 patients, the triplet arm showed a statistically significant improvement in mPFS (8.3 vs 5.7 months; HR 0.67; 95\% Cl 0.45-1.003; p 0.025) and ORR $(32.9 \%$ vs $13.9 \%, p$ 0.004) over the chemotherapy + trastuzumab arm, with a comparable safety profile, making of this combination an appealing option in the triple-positive disease. Despite these promising results, it must be noted that the third study arm combining abemaciclib plus trastuzumab did not show superiority over chemotherapy plus trastuzumab. Since there was no study arm with trastuzumab plus fulvestrant alone, the influence of abemaciclib to the observed PFS advantage remains unclear.

\section{Triple-negative breast cancer}

Historically, the denomination of TNBC used to imply the absence of known druggable targets for this subset of $\mathrm{BCs}$, which is the reason why this cancer's systemic treatment has mostly relied on chemotherapies for decades. Nonetheless, recent advancements in drug development lead to the approval of the first immunotherapy for PDL1-positive advanced TNBC and two oral PARP-inhibitors for gBRCAmutated $A B C$ s. Moreover, several other targeted agents are demonstrating activity in this subtype of $B C$, challenging the classification itself. In this context, one emerging entity is HER2-low expressing TNBCs, for which various drugs are showing encouraging activity, as well as TNBC expressing TROP2, LIV1, and other targetable antigens.

\subsection{Immune checkpoint inhibitors}

Several anti-PD(L)1 antibodies have shown various degrees of activity in advanced TNBC. In particular, higher activity has been described in first-line treatment, in combination with chemotherapy and in patients expressing PD-L1 on tumorinfiltrating immune cells [63]. For the purpose of this review, due to its recent approval, only atezolizumab will be reviewed. 
EXPERT OPINION ON BIOLOGICAL THERAPY

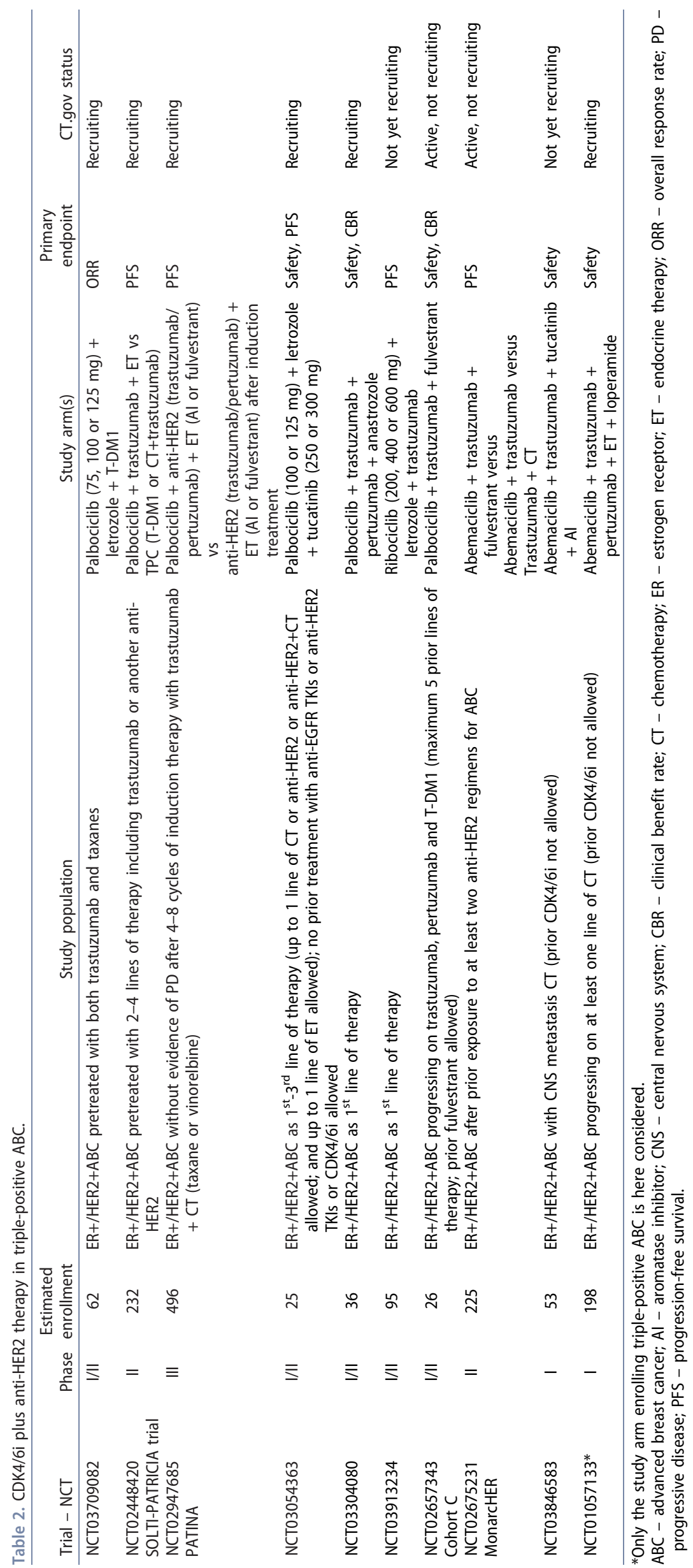


Nonetheless, we have data available on the activity of several other anti-PD-(L)1 agents, including nivolumab, avelumab, durvalumab, and pembrolizumab [64]. Moreover, for the latter agent, a randomized phase 3 trial is ongoing to determine its activity in combination with chemotherapy.

Atezolizumab is an anti-PD-L1 antibody able to prevent PDL1 interaction with the receptors PD-1 and B7-1, reversing T-cell suppression. After demonstrating a good safety profile and a variable activity in early phase trials, the large phase 3 IMpassion130 trial was initiated, randomizing 451 advanced TNBC patients to receive nab-paclitaxel with atezolizumab or placebo as first-line treatment [65]. The combination ultimately showed a statistically significant prolongation of PFS, both in PD-L1-positive patients (7.5 vs 5 months; HR 0.62; 95\% $\mathrm{Cl}$ 0.49-0.78; $\mathrm{p}<0.001)$ and in the intention-to-treat population (7.2 vs 5.5 months, HR 0.80; 95\% Cl 0.69-0.92; p 0.002). Due to the design of the trial, no statistically significant OS benefit could be proven; nonetheless, a numerical advantage of 7 months [66] was reported in PD-L1 positive patients. The combination was well tolerated, with a $49 \%$ G3-4 AE rate (vs $42 \%$ in the control arm). Based on these results, the regimen was approved as first-line treatment of advanced PDL1positive TNBC, becoming the first immunotherapy approval for the treatment of BC [67]. Numerous trials are testing other atezolizumab combinations in the same setting, in order to determine the best regimen in first-line treatment of advanced TNBC. As an example, preliminary results from a phase $1 b$ trial testing the triplet of a taxane + atezolizumab + ipatasertib (AKT inhibitor) demonstrated an impressive 73\% ORR irrespective of PD-L1 status, with a manageable toxicity [68]. Data regarding further combinations are awaited.

A particular mention is needed for immune induction strategies in advanced TNBC. A recent report from the ongoing phase 2 TONIC trial showed interesting clinical and translational outcomes of a 2-week low-dose induction with chemotherapy (cisplatin, doxorubicin, or cyclophosphamide) or radiotherapy before starting immunotherapy with the antiPD1 agent nivolumab [69]. In particular, the highest response rates were obtained with cisplatin (23\%) and doxorubicin (35\%) induction, as well as an upregulation of immunerelated genes involved in PD-L1 and T cell cytotoxicity pathways in the same cohorts.

\subsection{PARP-inhibitors}

Since the first preclinical reports, the sensitivity of BRCA1- and BRCA2-mutant BC cells to PARP-inhibitors has been extensively studied, leading to the development of multiple PARP-inhibitors [70]. These inhibitors vary in their activity and toxicity, mostly due to their PARP trapping potency. Two PARP-inhibitors are currently approved for the treatment of gBRCA-mut HER2negative $B C$, regardless of hormone receptors expression.

Olaparib is an oral PARP-inhibitor with an average PARP trapping potency. The compound was compared to standard chemotherapy (capecitabine, eribulin, or vinorelbine) in the phase 3 OlympiAD trial, enrolling gBRCAmut HER2-negative $A B C$ patients [71]. The trial demonstrated a PFS benefit for the olaparib arm (7 vs 4.2 months; HR $0.58 ; 95 \%$ Cl $0.43-0.80$; $p<0.001$ ) as well as a higher response rate (ORR $60 \%$ vs
$29 \%)$, with a better toxicity profile compared to chemotherapy. Most common AEs were hematological and gastrointestinal, more commonly G1-2. Based on these data, olaparib received FDA-approval for the treatment of gBRCA-mut HER2negative $A B C$ who have been previously treated with chemotherapy.

Talazoparib is an oral PARP-inhibitor with a high PARP trapping potency, about 100 times greater than that of olaparib [72]. Talazoparib was compared to standard chemotherapy (capecitabine, eribulin, gemcitabine, or vinorelbine) in the phase 3 EMBRACA trial, for gBRCA-mut HER2-negative $A B C$ patients [73]. Patients in the talazoparib arm experienced a significantly longer mPFS (8.6 vs. 5.6 months; HR $0.54 ; 95 \%$ $\mathrm{Cl} 0.41-0.71 ; \mathrm{p}<0.001)$ and response rate (ORR $62.6 \%$ vs $27 \%$, $p<0.001)$ compared with the control arm. However, this was achieved at the expense of a higher toxicity rate, mostly hematological, with $55 \%$ of the patients experiencing G3-4 hematological AEs in the talazoparib arm compared with $38 \%$ in the control arm. Following these results, talazoparib was approved by FDA for the treatment of germline BRCAmutated HER2-negative $A B C$.

Interestingly, data on the combination of PARP-inhibitors and immunotherapy with anti-PD(L)1 mAbs are emerging. In the phase 2 MEDIOLA trial, 34 gBRCA-mut HER2-negative ABC patients were treated with olaparib and durvalumab [74]. Of the 30 patients evaluable for response, 19 achieved a response, for an ORR or $63 \%$, and a mPFS of 8.2 months. Of note, response rate tended to be higher in less pretreated patients, consistently with other immunotherapy trials in $A B C$. Slightly different was the design of the phase 2 TOPACIO trial, which tested the combination of niraparib and pembrolizumab in patients with advanced TNBC, irrespective of BRCA mutation status [75]. Preliminary results were recently reported and showed an ORR of $11 \%$ in BRCA-wild type patients, which raised to $47 \%$ in BRCA-mutant patients. The combination was tolerable, with mostly hematological AEs.

Finally, results from a randomized phase 3 trial testing the combination of the PARP-inhibitor veliparib with carboplatin and paclitaxel in gBRCA-mut $A B C$ patients were recently presented [76]. Veliparib showed to significantly improve mPFS (14.5 vs 12.6 months, $p=0.002$ ), with comparable AEs. Nevertheless, it must be noted that the study allowed for a maintenance therapy with veliparib in absence of disease progression, so it is not clear if the combined therapy with chemotherapy is needed for this PFS improvement. ORR and OS were not significantly different in the two arms.

Although not immediately practice changing, these data confirm the feasibility of combining PARP-inhibitors with chemotherapy, despite their overlapping toxicity profile.

\subsection{Antibody-drug conjugates}

Beside ADCs targeting low HER2 expressions, novel ADCs against several other targets are being investigated in TNBC. Differently from HER2, such targets are not necessarily involved in oncogenic pathways, since the main anti-tumoral activity is provided by the chemotherapy payload carried by the ADC. Target antigens need instead to be tumor-specific (or 
tumor-associated) and have high levels of expression and penetrance in tumor cells.

Sacituzumab Govitecan-hziy is ADC coupling the topoisomerase I inhibitor SN-38 to a humanized anti-trophoblast cell-surface antigen 2 (Trop-2) mAb through a cleavable linker. Trop- 2 is detected in BC cells, including TNBC, and its expression is reported in more than $85 \%$ of tumors. In a phase $1 / 2$ trial enrolling 108 advanced TNBC patients, the compound showed to be safe, with few G3-4 AEs. The most common adverse events were nausea, diarrhea, fatigue, neutropenia, and anemia. The ORR was 33\%, with a median duration of responses of 7.7 months, a mPFS of 5.5 months and a median OS of 13 months [77]. The randomized phase 3 ASCENT trial is currently comparing sacituzumab govitecan-hziy with the physician's choice of single-agent chemotherapy in advanced TNBC (NCT02574455).

Ladiratuzumab Vedotin is an anti-LIV-1 antibody conjugated via a cleavable linker to an auristatin payload. LIV-1 is a transmembrane protein expressed in about $90 \%$ of $A B C$, with a much lower expression in normal tissues. In a phase 1 trial, 63 advanced TNBC patients received ladiratuzumab vedotin; LIV-1 tumor expression was required for the enrollment [78]. The compound was safe and well tolerated, with most of G3-4 AEs being hematological; of note, all-grade alopecia and peripheral neuropathy were reported in $36 \%$ and $20 \%$ of the patients, respectively. An ORR of $25 \%$ was achieved, with a DCR of $35 \%$. Various trials testing the compound in monotherapy and in different combinations are currently ongoing.

Additional ADCs for which encouraging early results were reported are: the anti-PTK7 ADC PF-7020, which showed an ORR of $21 \%$ in pretreated TNBC patients in a phase 1 trial [79]; the anti-EFNA4 ADC PF-06647263, showing a 10\% ORR in BC patients in a phase 1 trial [80]; the anti-gpNMB ADC glembatumumab vedotin, which showed an ORR of $16 \%$ (vs $15 \%$ with capecitabine) in gpNMB-overexpressing TNBC in a randomized phase $2 b$ trial [81]. Several other ADCs are under investigation in both HER2-positive and TNBC (Table 3).

\section{Future directions}

A variety of novel biologic treatment approaches are being investigated in the treatment of all subtypes of $A B C$ (Figure 2). In the next paragraphs, some recent advancements in $B C$ immunotherapy and targeted therapy are discussed.

\subsection{Immunotherapy beyond ICPI: adoptive cell therapy}

The term 'adoptive cell therapy' refers to a relatively new immunotherapy technique based on adoptive transfer of $T$ cells engineered ex-vivo to have chimeric antigen receptors (CAR) or tumor-infiltrating lymphocytes (TILs) targeting tumor antigens. Few pieces of evidence are available about the efficacy of these treatments in solid tumors, including BC.

CAR-T cells are genetically modified autologous T-cells that present on their surface chimeric receptors specific for tumorassociated antigens (TAAs), along with various costimulatory molecules.

HER2, mesothelin, and ROR1 are the main TAAs under investigation for CAR-T therapy. HER2- and mesothelin- targeted CAR-T showed efficacy in both in vitro cell lines and mice models [82-85], but at present few evidences [86,87] are available about their activity in patients with $A B C$. Some early clinical trials recruiting also $B C$ patients are ongoing (NCT02792114, NCT03740256, NCT03198052, NCT03696030, NCT03747965, NCT03545815, NCT03615313). ROR1-targeted CAR-T cells are also being tested in clinical trials, and a preliminary report on 4 TNBC patients showed the treatment to be safe and potentially active [88].

Even if the rational is strong, many questions need to be solved. The major challenge associated to CAR-T therapy is related to the rarity of 'real' tumor antigens, and consequently to the 'on-target, off-tumor' toxicity associated to antigen expression in normal tissues [89]. Moreover, TAAs are frequently subjected to immune escape, a well-described mechanism of resistance that consists of antigenic shift and production of new antigens no more recognized by specific CAR-T cells. Issues related to limited survival of the CAR-T cells, to their inefficient homing and to resistance to immunesuppressive tumor microenvironment will also need to be addressed.

TILs therapy instead relies on isolation of antitumor $T$ lymphocytes infiltrating the tumor stroma, their expansion ex vivo and re-infusion. Before being re-infused, TILs are cocultured with autologous dendritic cells previously engineered to recognize specific tumor antigens selected by tumor DNA sequencing.

This strategy has demonstrated to be effective in tumors with high levels of mutations and high TILs, such as melanoma [90]. Thanks to advances in TILs isolation, identification of tumor mutations and new cellular engineering techniques, TILs therapy has recently achieved significant results also in epithelial cancers, including BC. Nevertheless, most of these results are presented as single case reports [91]. Designing and conduction of clinical trials for such complex and ultrapersonalized therapies is faced with many issues and costs, requiring unique expertise and laboratory infrastructures. A phase 2 clinical trial recruiting several advanced tumor types, including $A B C$, is ongoing at the National Cancer Institute (NCT01174121).

\subsection{Anti-HER3 agents}

Enhanced expression of HER3 has been reported in $50-70 \%$ of $\mathrm{BC}$, in which it seems to represent a poor prognostic factor. HER3-overexpression has been associated to endocrine resistance in luminal BC [92], and with poor sensitivity to anti-HER2 agents [93] in HER2-amplified BC.

Several anti-HER3 monoclonal and bispecific Abs have been developed in order to overcome this resistance. Patritumab (U31287/A888), Seribatumumab (MM-121), and Lumretuzumab (RG7116, RO-5479599) are all anti-HER3 mAb tested in BC.

In HER2-amplified $A B C$ the combination of patritumab plus trastuzumab and paclitaxel demonstrated to be safe and active, with an ORR of 38.9\% [94]. Seribantumab has been investigated both in combination with paclitaxel in HER2-negative $A B C$ and with $E T$ in luminal $A B C$. Data about the combination with exemestane are available [95], showing a favorable trend in prolonging PFS and a significant increased OS. Lumretuzumab has been 


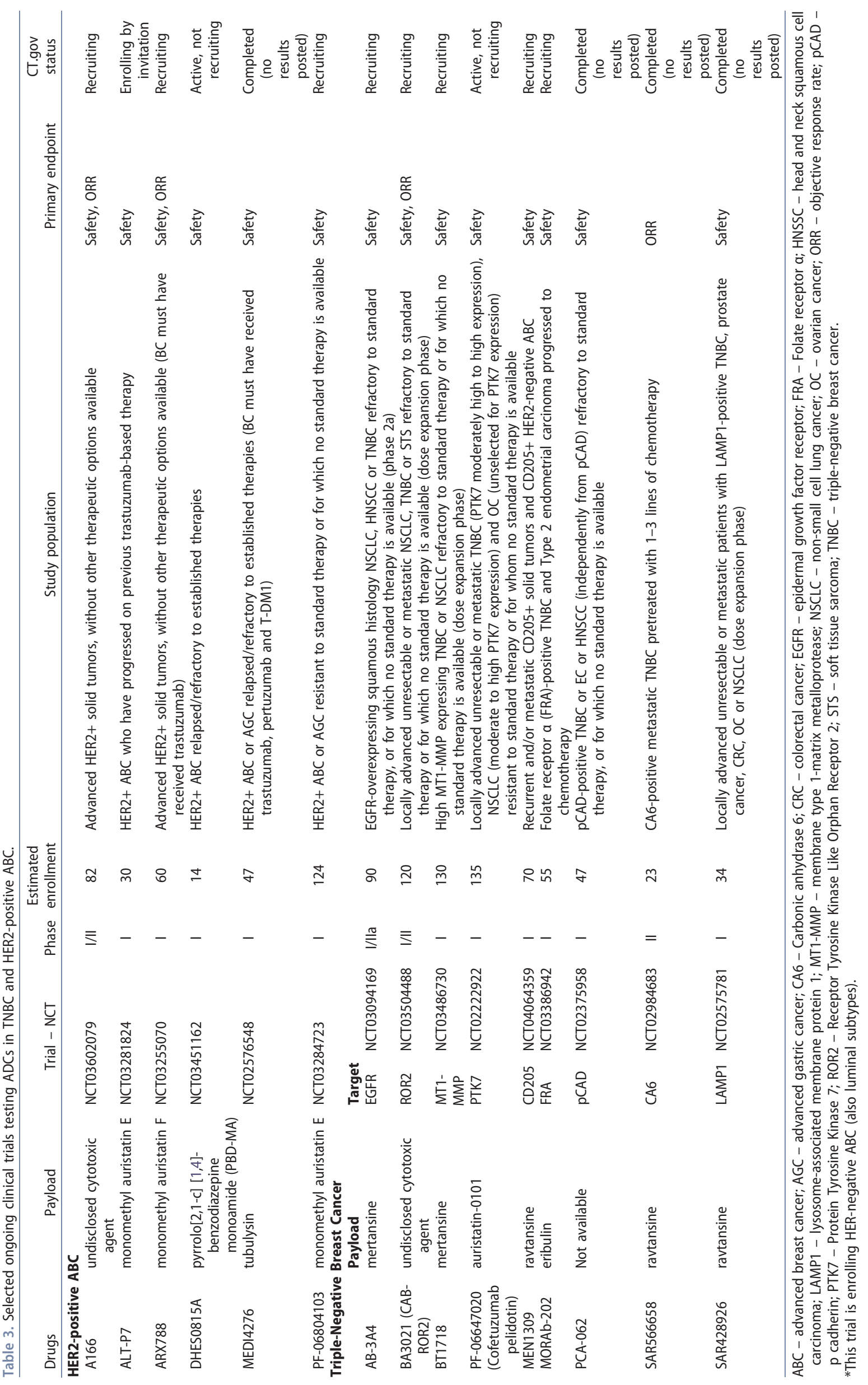




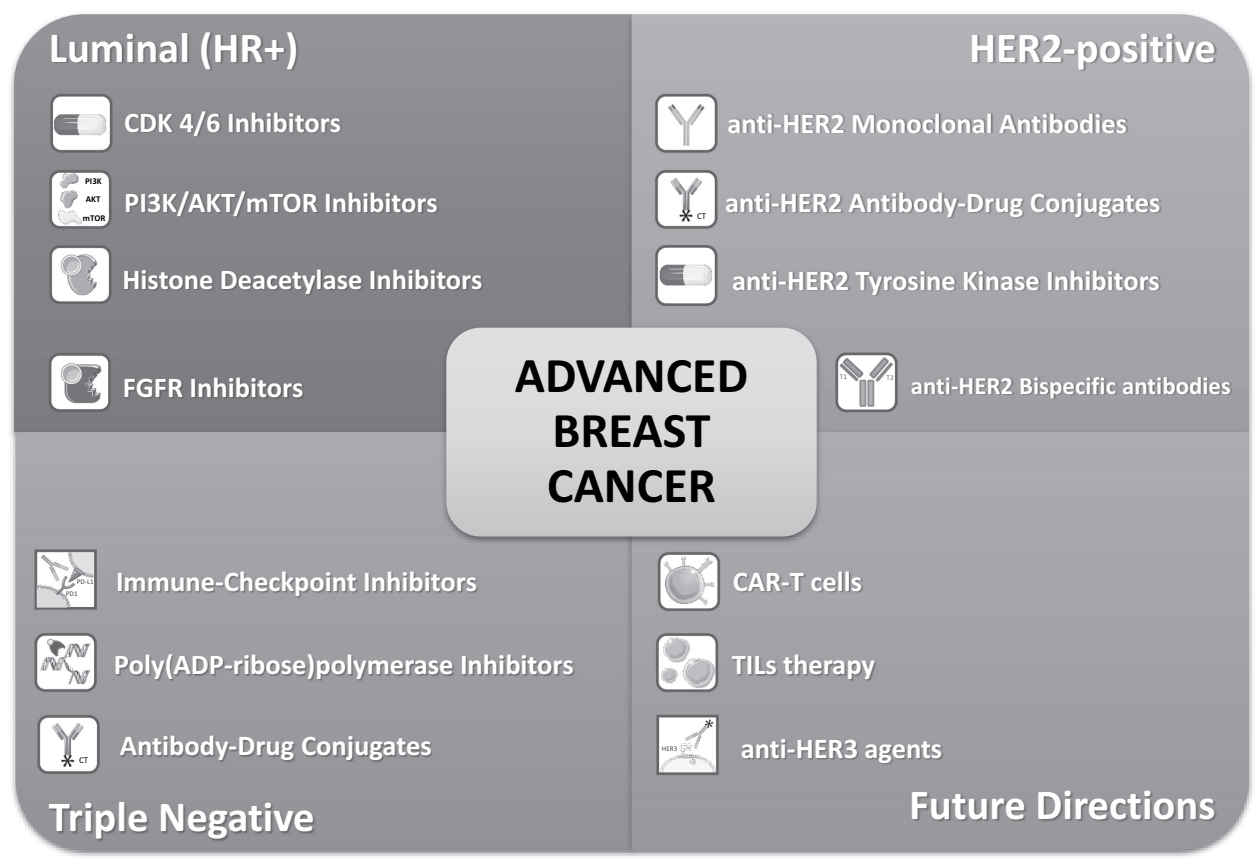

Figure 2. Latest advancements in advanced breast cancer biologic treatment.

All BC subtypes have seen several biologic drugs being approved for their treatment. Moreover, a variety of new biologic treatments are being tested and might enlarge the therapeutic arsenal of each subtype. This figure was created using Servier Medical Art templates, which are licensed under a Creative Commons Attribution 3.0 Unported License; https://smart.servier. com.

combined with paclitaxel and pertuzumab for the treatment of HER2-low/HER3 + ABC [96]. Observed ORR was high (55\% and $38.5 \%$ in different dose-cohorts), but the therapeutic window was too narrow to allow for further clinical development.

However, the most encouraging signals to date come from the phase $1 / 2$ study of U3-1402, an anti-HER3 ADC conjugated with a topoisomerase I inhibitor payload, with a high DAR (7:1 to 8:1). The compound was tested in 42 heavily pretreated HER3-positive (IHC score 2+/3+) ABC patients and showed a promising activity regardless of HER2-positivity [97]. In fact, only $16 \%$ of patients enrolled in the trial were HER2-positive, with the vast majority comprising hormone receptor-positive and TNBC patients. Forty-two patients were treated in the dose-expansion part of the trial, with an ORR of $42.9 \%$, a mDCR of $90.5 \%$, and a mPFS of 8.3 months. Antitumor activity was observed in all molecular subtypes, and treatment was moderately tolerated, with most common $\geq \mathrm{G} 3$ AEs being thrombocytopenia (35\%), neutropenia (28\%), leukopenia $(21 \%)$, and anemia (16\%).

\section{Expert opinion}

Significant advancements for the management of $A B C$ have been achieved, as a result of better understanding of the molecular mechanisms underlying breast oncogenesis and resistance-generation to treatments. Indeed, the last decade has seen a progressive increase in the development and approval of new biological drugs for the treatment of all $A B C$ subtypes, and today, in developed countries, most $A B C$ patients receive a biologic agent as first-line treatment for their disease.
However, the extent of clinical benefit provided by such agents is highly variable and needs to be constantly weighted against the possible increase in toxicities. For instance, in the setting of luminal $A B C$, several attempts to combine ET with biological agents have been made, with various compounds being approved for this indication. Some of these agents, such as CDK4/6-inhibitors, have rapidly acquired a preeminent role in all guidelines, due to the favorable safety profile. Some others, such as everolimus, are still only partially implemented in everyday practice, due to the less manageable toxicities. The same applies for other $A B C$ subtypes: for instance, while the first- and second-line treatments of HER2-positive $A B C$ are well defined, several drugs are currently approved for pretreated HER2-positive $A B C$, challenging clinicians in the choice of the most appropriate agent. In this framework, a promising tool may help to weight the clinical value of novel agents, namely the ESMO Magnitude of Clinical Benefit Scale (MCBS) [98], whose implementation could significantly help medical oncologists in the process of clinical decision-making.

Beside the relevant drug approvals in the recent past, a variety of novel compounds are showing encouraging results in all $A B C$ subtypes, and promise to further improve outcomes of these patients. While some of these drugs represent pharmacological evolutions of previously approved agents (e.g. novel anti-HER2 TKIs), some others might pave the way for new paradigms in $A B C$ treatment (Figure 3). In particular, novel ADCs are showing activity via the targeting of antigens which are not necessarily involved in oncogenic pathways. Key examples are trastuzumab deruxtecan and trastuzumab duocarmazine, showing activity in HER2-low non-amplified tumors, as well as Sacituzumab Govitecan, Ladiratuzumab Vedotin, and further ADCs targeting 


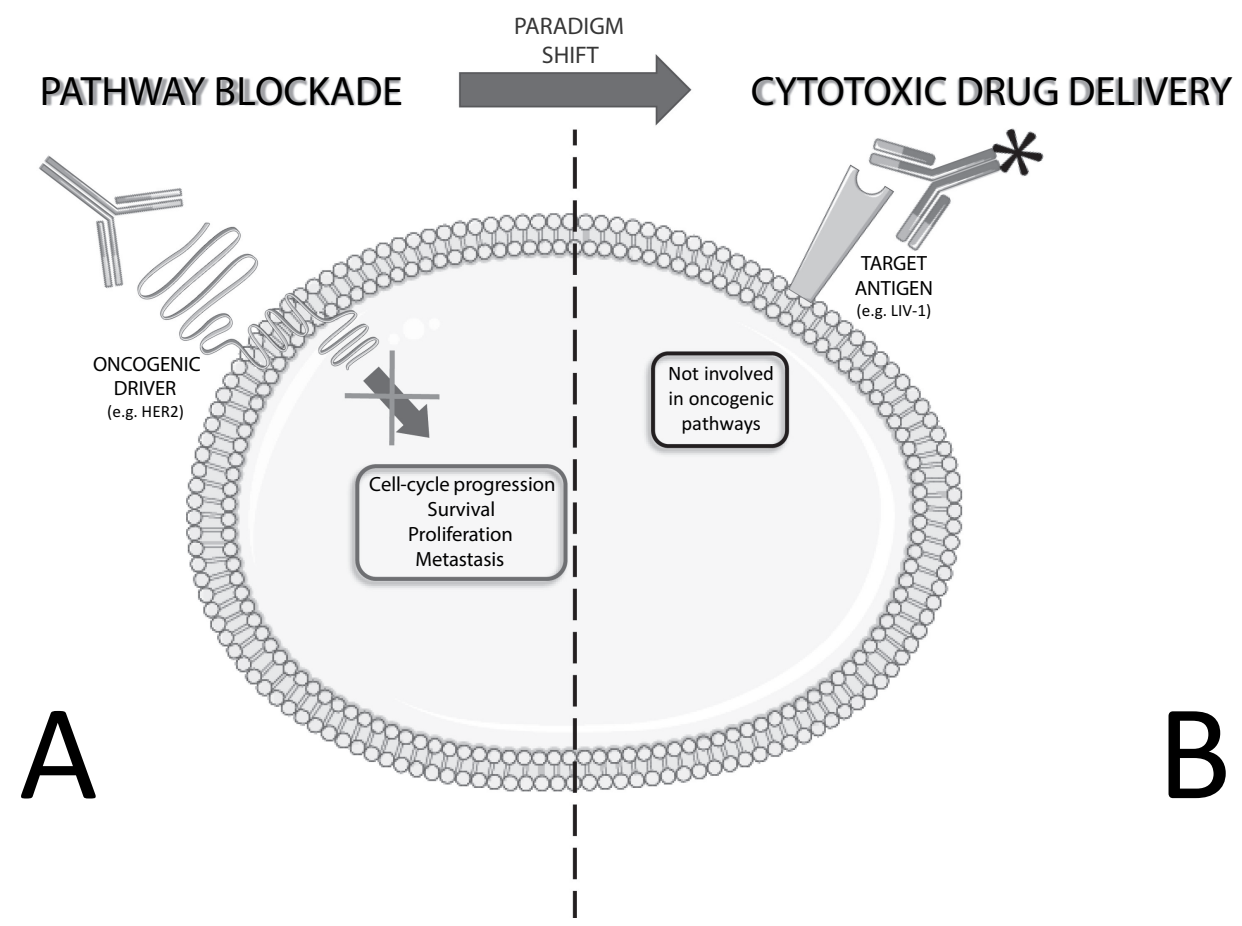

Figure 3. New paradigms in advanced breast cancer biologic treatment.

(a) The traditional paradigm in breast cancer biologic treatment involves the disruption of oncogenic pathways, which ultimately leads to an inhibition of cell survival, proliferation, and metastatization. (b) Novel antibody-drug conjugates allow the delivery of cytotoxic drugs to cancer cells, through the targeting of TAA which are not necessarily involved in oncogenic pathways. This figure was created using Servier Medical Art templates, which are licensed under a Creative Commons Attribution 3.0 Unported License; https://smart.servier.com.

TAA expressed by TNBC cells. If early results from these compounds are confirmed, a considerable evolution of $A B C$ treatment algorithms is expected.

Finally, the approval of biologic agents restricted to patients harboring predictive genetic alterations enhances the need to sequence $A B C$ to choose the best treatment strategy. While HER2 status once was the only genomic information to guide the treatment algorithm, many more alterations are gaining importance to predict drug efficacy, such as PIK3CA and BRCA status; some others are showing potential in predicting drug resistance, such as ESR1 and PTEN. More in detail, by applying the ranking from ESMO Scale for Clinical Actionability of molecular Targets (ESCAT scale), around 40 recurrent driver alterations are found in BC [99]. Some of these (ERBB2 amplifications, BRCA1/2 mutations, PIK3CA mutations) are classified tier of evidence IA, which implies a high level of actionability and a proven benefit observed in large randomized trials. Additionally, tumor-agnostic alterations like NTRK fusions and microsatellite instability are ranked tier IC and are expected to be actionable based on studies enrolling a wide variety of cancers. The growing number of useful biomarkers is promoting big changes in BC diagnostics, with multigene NGS panels being already applied in several countries to comprehensively capture the complexity of each tumor with a single test.

However, the escalating costs of both diagnostic assays and novel drugs might represent an obstacle in their implementation, in particular in the framework of developing countries, where huge disparities in healthcare availability already exist.
In this complex scenario, the key to ensure sustainability for patients is to ensure the implementation based on the intrinsic value of assays and drugs, to make sure that value for the money is fulfilled.

In conclusion, novel biologic drugs and treatment strategies are currently revolutionizing $A B C$ treatment algorithms, and an enlarging pipeline of promising agents is expected to provide increasing benefits to $\mathrm{BC}$ patients, as well as to promote the advancement of precision medicine in the treatment of $\mathrm{BC}$.

\section{Acknowledgments}

We thank Dario Trapani for supporting proofreading.

\section{Funding}

This paper is not funded.

\section{Declaration of interest}

G Curigliano received honoraria for speaker, consultancy or advisory rule from Roche, Pfizer, Novartis, Seattle Genetics, Lilly, Ellipses Pharma, Foundation Medicine, and Samsung. The authors have no other relevant affiliations or financial involvement with any organization or entity with a financial interest in or financial conflict with the subject matter or materials discussed in the manuscript apart from those disclosed.

\section{Reviewer Disclosures}

Peer reviewers on this manuscript have no relevant financial relationships or otherwise to disclose. 


\section{References}

Papers of special note have been highlighted as either of interest $(\cdot)$ or of considerable interest $(\cdot \cdot)$ to readers.

1. Goldenberg MM. Trastuzumab, a recombinant DNA-derived humanized monoclonal antibody, a novel agent for the treatment of metastatic breast cancer. Clin Ther. 1999;21:309-318.

2. Pondé N, Brandão M, El-hachem G, et al. Treatment of advanced HER2-positive breast cancer : 2018 and beyond. Cancer Treat Rev. 2018;67:10-20.

3. Slamon DJ, Neven P, Chia S, et al. Overall survival (OS) results of the phase III MONALEESA-3 trial of postmenopausal patients (pts) with hormone receptor-positive (HR1), human epidermal growth factor 2-negative (HER22) advanced breast cancer (ABC) treated with fulvestrant (FUL) 6 ribociclib. Ann Oncol. 2019;30.

4. Sledge GW, Toi M, PN J, et al. LBA6_PR MONARCH 2: overall survival of abemaciclib plus fulvestrant in patients with HR+, HER2advanced breast cancer. Ann Oncol. 2019;30. DOI:10.1093/ annonc/mdz394.006.

5. Turner NC, Slamon DJ, Ro J, et al. Overall survival with palbociclib and fulvestrant in advanced breast cancer. $N$ Engl J Med. 2018;379:1926-1936.

6. André F, Ciruelos E, Rubovszky G, et al. Alpelisib for PIK3CA mutated, hormone receptor-positive advanced breast cancer. N Engl J Med. 2019;380:1929-1940.

7. F.D.A. Drug Approvals and Databases. FDA approves alpelisib for metastatic breast cancer. 2019.

8. Jensen EV, Jordan VC. The estrogen receptor: A model for molecular medicine. Clin Cancer Res. 2003;9:1980-1989.

9. Cardoso F, Senkus E, Costa A, et al. 4th ESO-ESMO international consensus guidelines for advanced breast cancer ( $A B C$ 4). Ann Oncol. 2018;29:1634-1657.

10. Osborne CK, Schiff R. Mechanisms of endocrine resistance in breast cancer. Annu Rev Med. Internet]. 2011;62:233-247. Available from. ;: http://www.annualreviews.org/doi/10.1146/annurev-med $-070909-182917$

11. Hortobagyi GN, Stemmer SM, Burris HA, et al. Updated results from MONALEESA-2, a phase III trial of first-line ribociclib plus letrozole versus placebo plus letrozole in hormone receptor-positive, HER2-negative advanced breast cancer. Ann Oncol. 2018;29:15411547.

12. Hortobagyi GN, Stemmer SM, Burris HA, et al. Ribociclib as first-line therapy for HR-positive, advanced breast cancer. N Engl J Med. 2016;375:1738-1748.

13. Finn RS, Martin M, Rugo HS, et al. Palbociclib and letrozole in advanced breast cancer. N Engl J Med. 2016;375:1925-1936.

14. Rugo HS, Finn RS, Diéras $V$, et al. Palbociclib plus letrozole as first-line therapy in estrogen receptor-positive/human epidermal growth factor receptor 2-negative advanced breast cancer with extended follow-up. Breast Cancer Res Treat. 2019;174:719-729.

15. Goetz MP, Toi M, Campone M, et al. MONARCH 3: abemaciclib as initial therapy for advanced breast cancer. J Clin Oncol. 2017;35:3638-3646.

16. Johnston S, Martin M, Di Leo A, et al. MONARCH 3 final PFS: a randomized study of abemaciclib as initial therapy for advanced breast cancer. Npj Breast Cancer. 2019;5:5.

17. Cristofanili M, Turner NC, Bondarenko I, et al. Fulvestrant plus palbociclib versus fulvestrant plus placebo for treatment of hormone-receptor-positive, HER2-negative metastatic breast cancer that progressed on previous endocrine therapy (PALOMA-3): final analysis of the multicentre, double-blind, phase. Lancet Oncol. 2016;17:425-439.

18. Sledge GW, Toi $M$, Neven $P$, et al. MONARCH 2: abemaciclib in combination with fulvestrant in women with HR+/HER2- advanced breast cancer who had progressed while receiving endocrine therapy. J Clin Oncol. 2017;35:2875-2884.

19. Slamon DJ, Neven P, Chia S, et al. Phase III randomized study of ribociclib and fulvestrant in hormone receptor-positive, human epidermal growth factor receptor 2-negative advanced breast cancer: MONALEESA-3. J Clin Oncol. 2018;36:2465-2472.
20. Im S-A, Lu Y-S, Bardia A, et al. Overall survival with ribociclib plus endocrine therapy in breast cancer. $N$ Engl J Med. 2019;381:307-316.

21. Slamon DJ, Neven P, Chia S, et al. LBA7_PROverall survival (OS) results of the phase III MONALEESA-3 trial of postmenopausal patients (pts) with hormone receptor-positive $(\mathrm{HR}+)$, human epidermal growth factor 2-negative (HER2-) advanced breast cancer $(A B C)$ treated with fulvestrant (FUL) \pm r. Ann Oncol. 2019;30.

22. Marra A, Curigliano G. Are all cyclin-dependent kinases $4 / 6$ inhibitors created equal? Npj Breast Cancer. 2019;5:27.

23. Saini KS, Loi S, de Azambuja E, et al. Targeting the PI3K/AKT/mTOR and Raf/MEK/ERK pathways in the treatment of breast cancer. Cancer Treat Rev. 2013;39:935-946.

24. Baselga J, Campone M, Piccart M, et al. Everolimus in postmenopausal hormone-receptor-positive advanced breast cancer. N Engl J Med. 2012;366:520-529.

25. Baselga J, Dent SF, Cortés J, et al. Phase III study of taselisib $($ GDC-0032) + fulvestrant (FULV) v FULV in patients (pts) with estrogen receptor (ER)-positive, PIK3CA -mutant (MUT), locally advanced or metastatic breast cancer (MBC): primary analysis from SANDPIPER. J Clin Oncol. 2018;36:LBA1006-LBA1006.

26. Baselga J, Im S-A, Iwata $H$, et al. Buparlisib plus fulvestrant versus placebo plus fulvestrant in postmenopausal, hormone receptor-positive, HER2-negative, advanced breast cancer (BELLE-2): a randomised, double-blind, placebo-controlled, phase 3 trial. Lancet Oncol. 2017;18:904-916.

27. Campone M, Im S-A, Iwata $H$, et al. Buparlisib plus fulvestrant versus placebo plus fulvestrant for postmenopausal, hormone receptor-positive, human epidermal growth factor receptor 2-negative, advanced breast cancer: overall survival results from BELLE-2. Eur J Cancer. 2018;103:147-154.

28. Di Leo A, Johnston S, Lee KS, et al. Buparlisib plus fulvestrant in postmenopausal women with hormone-receptor-positive, HER2-negative, advanced breast cancer progressing on or after mTOR inhibition (BELLE-3): a randomised, double-blind, placebo-controlled, phase 3 trial. Lancet Oncol. 2018;19:87-100.

29. Jones RH, Casbard A, Carucci M, et al. Fulvestrant plus capivasertib versus placebo after relapse or progression on an aromatase inhibitor in metastatic, oestrogen receptor-positive breast cancer (FAKTION): a multicentre, randomised, controlled, phase 2 trial. Lancet Oncol. 2020;21:345-357.

30. Connolly R, Stearns V. Epigenetics as a therapeutic target in breast cancer. J Mammary Gland Biol Neoplasia. 2012;17:191-204.

31. Yardley DA, Ismail-Khan R, Klein P. Results of ENCORE 301 , a randomized, phase II, double-blind, placebo-controlled study of exemestane with or without entinostat in postmenopausal women with locally recurrent or metastatic estrogen receptor-positive (ER + ) breast cancer progressing on a nonsteroidal aromatase inhibitor (Al). J Clin Oncol. 2011;29:268

32. Jiang Z, Li W, Hu X, et al. Tucidinostat plus exemestane for postmenopausal patients with advanced, hormone receptor-positive breast cancer (ACE): a randomised, double-blind, placebo-controlled, phase 3 trial. Lancet Oncol. 2019;20:806-815.

33. Perez-Garcia J, Muñoz-Couselo E, Soberino J, et al. Targeting FGFR pathway in breast cancer. Breast. 2018;37:126-133.

34. Seckl M, Badman PD, Liu X, et al. RADICAL trial: A phase Ib/lla study to assess the safety and efficacy of AZD4547 in combination with either anastrozole or letrozole in ER positive breast cancer patients progressing on these aromatase inhibitors (Als). J Clin Oncol. 2017;35:1059.

35. Campone M, Bachelot T, Penault-Llorca $F$, et al. A phase Ib dose allocation study of oral administration of lucitanib given in combination with fulvestrant in patients with estrogen receptor-positive and FGFR1-amplified or non-amplified metastatic breast cancer. Cancer Chemother Pharmacol. 2019;83:743-753.

36. Hui R, Pearson A, Cortés J, et al. Lucitanib for the treatment of HR +/HER2 - metastatic breast cancer: results from the multicohort phase II FINESSE study. Clin Cancer Res. 2019. clincanres.1164.2019.

37. Rugo HS, Im S-A, Wright GLS, et al. SOPHIA primary analysis: A phase 3 (P3) study of margetuximab (M) + chemotherapy (C)

Q5

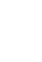


versus trastuzumab $(\mathrm{T})+\mathrm{C}$ in patients (pts) with HER2+ metastatic (met) breast cancer (MBC) after prior anti-HER2 therapies (Tx). J Clin Oncol. 2019;37:1000.

38. Rugo HS, Im S-A, Cardoso F, et al. Yardl and SSG. Abstract GS1-02: phase 3 SOPHIA study of margetuximab + chemotherapy vs trastuzumab + chemotherapy in patients with HER2 + metastatic breast cancer after prior anti-HER2 therapies: second interim overall survival analysis. SABCS. 2019;2019.

39. Martin $M$, Holmes FA, Ejlertsen $B$, et al. Neratinib after trastuzumab-based adjuvant therapy in HER2-positive breast cancer (ExteNET): 5-year analysis of a randomised, double-blind, placebo-controlled, phase 3 trial. Lancet Oncol. 2017;18:1688-1700.

40. Awada A, Colomer R, Inoue K, et al. Neratinib plus paclitaxel vs trastuzumab plus paclitaxel in previously untreated metastatic ERBB2-positive breast cancer. JAMA Oncol. 2016;2:1557.

41. Freedman RA, Gelman RS, Anders CK, et al. TBCRC 022: a phase II trial of neratinib and capecitabine for patients with human epidermal growth factor receptor 2-positive breast cancer and brain metastases. J Clin Oncol. 2019;37:1081-1089.

42. Saura C, Oliveira M, Feng $\mathrm{Y}-\mathrm{H}$, et al. Neratinib + capecitabine versus lapatinib + capecitabine in patients with HER2+ metastatic breast cancer previously treated with $\geq 2$ HER2-directed regimens: findings from the multinational, randomized, phase III NALA trial. J Clin Oncol. 2019;37:1002.

43. Borges VF, Ferrario C, Aucoin N, et al. Tucatinib combined with ado-trastuzumab emtansine in advanced ERBB2/HER2 -positive metastatic breast cancer. JAMA Oncol. 2018;4:1214.

44. Murthy R, Borges VF, Conlin A, et al. Tucatinib with capecitabine and trastuzumab in advanced HER2-positive metastatic breast cancer with and without brain metastases: a non-randomised, open-label, phase 1b study. Lancet Oncol. 2018;19:880-888.

45. Paplomata E, Bachelot T, Mueller V, et al. 198TiPA randomized, double-blinded, controlled study of tucatinib (ONT-380) vs placebo in combination with capecitabine $(C)$ and trastuzumab $(T)$ in patients with pretreated HER2+ unresectable locally advanced or metastatic breast carcinoma (mBC) (HER2CLIMB. Ann Oncol. 2019;30.

46. Murthy RK, Loi S, Okines A, et al. Tucatinib, trastuzumab, and capecitabine for HER2-positive metastatic breast cancer. N Engl J Med. 2019;NEJMoa1914609.

.. Of considerable interest due to the activity of the compound in the relevant unmet need of HER2-positive breast cancer patients with brain metastasis.

47. Park $\mathrm{YH}$, Lee $\mathrm{K}-\mathrm{H}$, Sohn $\mathrm{JH}$, et al. A phase II trial of the pan-HER inhibitor poziotinib, in patients with HER2-positive metastatic breast cancer who had received at least two prior HER2-directed regimens: results of the NOV120101-203 trial. Int J Cancer. 2018;143:3240-3247.

48. Jiang Z, Yan M, Hu X, et al. Pyrotinib combined with capecitabine in women with HER2+ metastatic breast cancer previously treated with trastuzumab and taxanes: A randomized phase III study. J Clin Oncol. 2019;37:1001.

49. Ma F, Ouyang Q, Li W, et al. Pyrotinib or lapatinib combined with capecitabine in HER2-positive metastatic breast cancer with prior taxanes, anthracyclines, and/or trastuzumab: a randomized, phase II study. J Clin Oncol. 2019;37:2610-2619.

50. Modi S, Saura C, Yamashita T, et al. Trastuzumab deruxtecan in previously treated HER2-positive breast cancer. N Engl J Med. 2019.

.. Of considerable interest due to the unprecedented activity demonstrated by the compound in highly pretreated HER2positive breast cancer patients.

51. Modi S, Park H, Murthy RK, et al. Antitumor activity and safety of trastuzumab deruxtecan in patients with HER2-low-expressing advanced breast cancer: results from a phase lb study. J Clin Oncol. 2020. JCO.19.02318. DOI:10.1200/JCO.19.02318.

.- Of considerable interest due to the activity of the drug in the emerging subgroup of HER2-low breast cancer.

52. Modi S, Ohtani S, Lee CC, et al. A phase III, multicenter, randomized, open label trial of [fam-] trastuzumab deruxtecan (DS-8201a) versus investigator's choice in HER2-low breast cancer. J Clin Oncol. 2019;37:TPS1102-TPS1102.

53. Banerji U, van Herpen CML, Saura C, et al. Trastuzumab duocarmazine in locally advanced and metastatic solid tumours and HER2-expressing breast cancer: a phase 1 dose-escalation and dose-expansion study. Lancet Oncol. 2019;2045:1-12.

- of considerable interest due to the activity of the compound in the emerging subgroup of HER2-low breast cancer

54. Saura C, Thistlethwaite F, Banerji U, et al. A phase I expansion cohorts study of SYD985 in heavily pretreated patients with HER2-positive or HER2-low metastatic breast cancer. J Clin Oncol. 2018;36:1014.

55. Meric-Bernstam F, Beeram M, Mayordomo Jl, et al. Single agent activity of ZW25, a HER2-targeted bispecific antibody, in heavily pretreated HER2-expressing cancers. J Clin Oncol. 2018;36:2500.

56. Loi S, Giobbie-Hurder A, Gombos A, et al. Pembrolizumab plus trastuzumab in trastuzumab-resistant, advanced, HER2-positive breast cancer (PANACEA): a single-arm, multicentre, phase 1b-2 trial. Lancet Oncol. 2019;20:371-382.

57. Emens L, Esteva F, Beresford M, et al. Abstract PD3-01: results from KATE2, a randomized phase 2 study of atezolizumab (atezo)+trastuzumab emtansine (T-DM1) vs placebo (pbo)+T-DM1 in previously treated HER2+ advanced breast cancer (BC). Poster Discuss. Abstr. American Association for Cancer Research; 2019. p. PD3-01.

58. Emens LA, Esteva FJ, Beresford $M$, et al. 30500verall survival (OS) in KATE2, a phase II study of programmed death ligand 1 (PD-L1) inhibitor atezolizumab (atezo)+trastuzumab emtansine (T-DM1) vs placebo (pbo)+T-DM1 in previously treated HER2+ advanced breast cancer (BC). Ann Oncol. 2019;30.

59. Witkiewicz AK, Cox D, Knudsen ES. CDK4/6 inhibition provides a potent adjunct to Her2-targeted therapies in preclinical breast cancer models. Genes Cancer. 2014;5:261-272.

60. Goel S, Wang $Q$, Watt AC, et al. Overcoming therapeutic resistance in HER2-positive breast cancers with CDK4/6 inhibitors. Cancer Cell. 2016;29:255-269.

61. Ciruelos E, Villagrasa P, Paré L, et al. Abstract PD3-03: SOLTI-1303 PATRICIA phase II trial (STAGE 1) - palbociclib and trastuzumab in postmenopausal patients with HER2-positive metastatic breast cancer; 2019, p. PD3-03.

62. Tolaney SM, Wardley AM, Zambelli $S$, et al. monarcHER: a randomized phase 2 study of abemaciclib plus trastuzumab with or without fulvestrant versus trastuzumab plus standard of care chemotherapy in women with HR+, HER2+ advanced breast cancer (ABC). Ann Oncol. 2019;30:v851-v934.

63. Tarantino P, Curigliano G. Defining the immunogram of breast cancer: a focus on clinical trials. Expert Opin Biol Ther. 2019;19:383-385.

64. Adams S, Gatti-Mays ME, Kalinsky K, et al. Current landscape of immunotherapy in breast cancer. JAMA Oncol. 2019;5:1205.

65. Schmid P, Adams S, Rugo HS, et al. Atezolizumab and Nab-paclitaxel in advanced triple-negative breast cancer. $\mathrm{N}$ Engl J Med. 2018;379:2108-2121.

66. Schmid P, Adams S, Rugo HS, et al. IMpassion130: updated overall survival (OS) from a global, randomized, double-blind, placebo-controlled, Phase III study of atezolizumab (atezo) + nabpaclitaxel (nP) in previously untreated locally advanced or metastatic triple-negative breast cancer (m. J Clin Oncol. 2019;37:1003.

- Of considerable interest due to its practice changing impact in the field of advanced triple-negative breast cancer.

67. F.D.A. Drug Approvals and Databases. FDA approves atezolizumab for PD-L1 positive unresectable locally advanced or metastatic triple-negative breast cancer; 2019 [cited 2019 Oct 14]. Available from: https://www.accessdata.fda.gov/drugsatfda_docs/label/2019/ 761034s018lbl.pdf

68. Schmid P, Loirat D, Savas P, et al. Abstract CT049: phase lb study evaluating a triplet combination of ipatasertib (IPAT), atezolizumab (atezo), and paclitaxel (PAC) or nab-PAC as first-line (1L) therapy for locally advanced/metastatic triple-negative breast cancer (TNBC). Clin Trials. American Association for Cancer Research. 2019:СT049-СT049. 
69. Voorwerk L, Slagter M, Horlings HM, et al. Immune induction strategies in metastatic triple-negative breast cancer to enhance the sensitivity to PD-1 blockade: the TONIC trial. Nat Med. 2019;25:920-928.

70. Mateo J, Lord CJ, Serra V, et al. A decade of clinical development of PARP inhibitors in perspective. Ann Oncol. 2019;30:1437-1447.

71. Robson M, Im S-A, Senkus E, et al. Olaparib for metastatic breast cancer in patients with a germline BRCA mutation. N Engl J Med. 2017;377:523-533.

72. Murai J, Huang S-YN, Renaud A, et al. Stereospecific PARP trapping by BMN 673 and comparison with olaparib and rucaparib. Mol Cancer Ther. 2014;13:433-443.

73. Litton JK, Rugo HS, Ettl J, et al. Talazoparib in patients with advanced breast cancer and a germline BRCA mutation. $\mathrm{N}$ Engl J Med. 2018;379:753-763.

74. Domchek S, Postel-Vinay S, Im S-A, et al. 11910Phase II study of olaparib (O) and durvalumab (D) (MEDIOLA): updated results in patients (pts) with germline BRCA-mutated (gBRCAm) metastatic breast cancer (MBC). Ann Oncol. 2019;30.

75. Vinayak S, Tolaney SM, Schwartzberg L, et al. Open-label clinical trial of niraparib combined with pembrolizumab for treatment of advanced or metastatic triple-negative breast cancer. JAMA Oncol. 2019;5:1132.

76. Diéras VC, Han HS, Kaufman B, et al. LBA9Phase III study of veliparib with carboplatin and paclitaxel in HER2-negative advanced/metastatic gBRCA-associated breast cancer. Ann Oncol. 2019;30.

77. Bardia A, Mayer IA, Vahdat LT, et al. Sacituzumab govitecan-hziy in refractory metastatic triple-negative breast cancer. $\mathrm{N}$ Engl J Med. 2019:380:741-751.

78. Modi S, Pusztai L, Forero A, et al. Phase 1 study of the antibody-drug conjugate ladiratuzumab vedotin (SGN-LIV1A) in patients with heavily pretreated triple-negative metastatic breast cancer. SABCS. 2017. Abstract $n$.

79. Sachdev JC, Maitland ML, Sharma M, et al. PF-06647020 (PF-7020), an antibody-drug conjugate (ADC) targeting protein tyrosine kinase 7 (PTK7), in patients (pts) with advanced solid tumors: results of a phase I dose escalation and expansion study. J Clin Oncol. 2018;36:5565.

80. Garrido-Laguna I, Krop I, Burris HA, et al. First-in-human, phase I study of PF-06647263, an anti-EFNA4 calicheamicin antibody-drug conjugate, in patients with advanced solid tumors. Int J Cancer. 2019;145:1798-1808.

81. Vahdat L, Forero-Torres A, Schmid P, et al. Abstract P6-20-01: METRIC: $A$ randomized international phase $2 \mathrm{~b}$ study of the antibody-drug conjugate (ADC) glembatumumab vedotin (GV) in gpNMB-overexpressing, metastatic, triple-negative breast cancer (mTNBC). Poster Sess. Abstr. American Association for Cancer Research. 2019. p. P6-20-01.

82. Sun M, Shi H, Liu C, et al. Construction and evaluation of a novel humanized HER2-specific chimeric receptor. Breast Cancer Res. 2014;16:R61.

83. Morales JK, Kmieciak M, Graham L, et al. Adoptive transfer of HER2/ neu-specific $T$ cells expanded with alternating gamma chain cytokines mediate tumor regression when combined with the depletion of myeloid-derived suppressor cells. Cancer Immunol Immunother. 2009;58:941-953.

84. Wilkie S, van Schalkwyk MCl, Hobbs S, et al. Dual targeting of ErbB2 and MUC1 in breast cancer using chimeric antigen receptors engineered to provide complementary signaling. J Clin Immunol. 2012;32:1059-1070.
85. Globerson-Levin A, Waks T, Eshhar Z. Elimination of progressive mammary cancer by repeated administrations of chimeric antigen receptor-modified T cells. Mol Ther. 2014;22:1029-1038.

86. Disis ML, Coveler AL, Higgins D, et al. Phase I/II study of adoptive T-cell therapy following in vivo priming with a HER2/neu vaccine in patients with advanced-stage HER2 + breast cancer. J Clin Oncol. 2014;32:615.

87. Adusumilli PS, Zauderer MG, Rusch VW, et al. Regional delivery of mesothelin-targeted CAR T cells for pleural cancers: safety and preliminary efficacy in combination with anti-PD-1 agent. J Clin Oncol. 2019;37:2511.

88. Specht J, Lee S, Turtle C, et al. Abstract P2-09-13: A phase I study of adoptive immunotherapy for ROR1+ advanced triple negative breast cancer (TNBC) with defined subsets of autologous T cells expressing a ROR1-specific chimeric antigen receptor (ROR1-CAR). Poster Sess. Abstr. American Association for Cancer Research; 2019. p. P2-09-13.

89. Morgan RA, Yang JC, Kitano $M$, et al. Case report of a serious adverse event following the administration of $\mathrm{T}$ cells transduced with a chimeric antigen receptor recognizing ERBB2. Mol Ther. 2010;18:843-851.

90. Rohaan MW, van den Berg JH, Kvistborg P, et al. Adoptive transfer of tumor-infiltrating lymphocytes in melanoma: a viable treatment option. J Immunother Cancer. 2018;6.

91. Zacharakis $\mathrm{N}$, Chinnasamy $\mathrm{H}$, Black $\mathrm{M}$, et al. Immune recognition of somatic mutations leading to complete durable regression in metastatic breast cancer. Nat Med. 2018;24:724-730.

92. Frogne T, Benjaminsen RV, Sonne-Hansen $K$, et al. Activation of ErbB3, EGFR and Erk is essential for growth of human breast cancer cell lines with acquired resistance to fulvestrant. Breast Cancer Res Treat. 2009;114:263-275.

93. Huang X, Gao L, Wang S, et al. Heterotrimerization of the growth factor receptors erbB2, erbB3, and insulin-like growth factor-I receptor in breast cancer cells resistant to herceptin Cancer Res. 2010;70:1204-1214.

94. Mukai $H$, Saeki T, Aogi K, et al. Patritumab plus trastuzumab and paclitaxel in human epidermal growth factor receptor 2-overexpressing metastatic breast cancer. Cancer Sci. 2016;107:1465-1470.

95. Finn $G$, Zhang $H$, Blois $A$. A randomized trial of exemestane \pm seribantumab (MM-121) in postmenopausal women with locally advanced or metastatic ER/PR+ HER2- breast cancer: final analysis and extended subgroup analysis. AACR Precis Med. 2016.

96. Schneeweiss A, Park-Simon TW, Albanell J, et al. Phase lb study evaluating safety and clinical activity of the anti-HER3 antibody lumretuzumab combined with the anti-HER2 antibody pertuzumab and paclitaxel in HER3-positive, HER2-low metastatic breast cancer. Invest New Drugs. 2018;36:848-859.

97. Yonemori K, Masuda N, Takahashi S, et al. 1510 Single agent activity of U3-1402, a HER3-targeting antibody-drug conjugate, in HER3-overexpressing metastatic breast cancer: updated results from a phase I/II trial. Ann Oncol. 2019;30.

98. Cherny NI, Dafni U, Bogaerts J, et al. ESMO-magnitude of clinical benefit scale version 1.1. Ann Oncol. 2017;28:2340-2366.

99. Mateo J, Chakravarty D, Dienstmann R, et al. A framework to rank genomic alterations as targets for cancer precision medicine: the ESMO scale for clinical actionability of molecular targets (ESCAT). Ann Oncol. 2018:29:1895-1902. 